\title{
Quantum Imaging with Incoherent Photons
}

\author{
C. Thiel, ${ }^{1}$ T. Bastin, ${ }^{2}$ J. Martin, ${ }^{2}$ E. Solano, ${ }^{3,4}$ J. von Zanthier, ${ }^{1, *}$ and G. S. Agarwal ${ }^{5}$ \\ ${ }^{1}$ Institut für Optik, Information und Photonik, Max-Planck-Forschungsgruppe, Universität Erlangen-Nürnberg, \\ 91058 Erlangen, Germany \\ ${ }^{2}$ Institut de Physique Nucléaire, Atomique et de Spectroscopie, Université de Liège, 4000 Liège, Belgium \\ ${ }^{3}$ Physics Department, ASC, and CeNS, Ludwig-Maximilians-Universität, Theresienstrasse 37, 80333 Munich, Germany \\ ${ }^{4}$ Sección Física, Departamento de Ciencias, Pontificia Universidad Católica del Perú, Apartado Postal 1761, Lima, Peru \\ ${ }^{5}$ Department of Physics, Oklahoma State University, Stillwater, Oklahoma 74078, USA
}

(Received 27 July 2006; published 28 September 2007)

\begin{abstract}
We propose a technique to obtain subwavelength resolution in quantum imaging with potentially $100 \%$ contrast using incoherent light. Our method requires neither path-entangled number states nor multiphoton absorption. The scheme makes use of $N$ photons spontaneously emitted by $N$ atoms and registered by $N$ detectors. It is shown that for coincident detection at particular detector positions a resolution of $\lambda / N$ can be achieved.
\end{abstract}

DOI: $10.1103 /$ PhysRevLett.99.133603

PACS numbers: 42.50.St, 03.65.Ud, 42.30.- d, 42.50.Dv

In Young's double slit experiment (or in a MachZehnder interferometer) the probability $G^{(1)}(\mathbf{r})$ to detect a photon at position $\mathbf{r}$ results from the interference of the two possible paths a single photon can take to reach the detector. This is expressed by the state $|\psi(1)\rangle=$ $1 / \sqrt{2}\left(|1\rangle_{U}|0\rangle_{L}+|0\rangle_{U}|1\rangle_{L}\right)$, where the subscript $L(U)$ denotes the lower (upper) arm of the interferometer. Variation of the detector position leads to a modulation of the form $G^{(1)}(\mathbf{r}) \propto 1+\cos \delta(\mathbf{r})$, where $\delta(\mathbf{r})=k d \sin \theta(\mathbf{r})$ is the optical phase difference of the waves emanating from the two slits and $k, d$, and $\theta(\mathbf{r})$ are the wave number, slit separation, and scattering angle, respectively. Obviously, the fringe spacing of the modulation [in units of $d \sin \theta(\mathbf{r})]$ is determined by the optical wavelength $\lambda$, in correspondence with the Rayleigh criterion [1].

Quantum entanglement is able to bypass the Rayleigh limit [2-11]. Consider, for example, the path-entangled $N$-photon state $|\psi(N)\rangle=1 / \sqrt{2}\left(|N\rangle_{U}|0\rangle_{L}+|0\rangle_{U}|N\rangle_{L}\right)$. Because the $N$-photon state $|N\rangle$ has $N$ times the energy of the single-photon state $|1\rangle$ in a given mode it accumulates phase $N$ times as fast when propagating through the setup. This gives rise to an $N$-photon absorption rate of the form $G^{(N)}(\mathbf{r}, \ldots, \mathbf{r}) \propto 1+\cos N \delta(\mathbf{r})$ exhibiting a fringe spacing $N$ times narrower than that of $G^{(1)}(\mathbf{r})$ [4]. This gain in resolution can be fruitfully applied to a wide range of applications, e.g., to lithography [4,5], microscopy [8], spectroscopy [9], and even magnetometry [10]. In order to implement this $N$-fold increase in resolution commonly an entangled state of the form $|\psi(N)\rangle$ in combination with a nonlinear medium sensitive to $\mathrm{N}$-photon absorption is needed [11].

In this Letter we propose a different scheme to achieve a resolution of $\lambda / N$ involving neither of the above requirements. In what follows we will apply this scheme in the context of microscopy. The method employs $N$ photons spontaneously emitted from $N$ atoms subsequently de- tected by $N$ detectors where by means of post-selection it is ensured that precisely one photon is recorded at each of the $N$ detectors. We demonstrate that in this case, for certain detector positions $\mathbf{r}_{2}, \ldots, \mathbf{r}_{N}$, the $N$ th order correlation function as a function of $\mathbf{r}_{1}$ takes the form $1+$ $\cos N \delta\left(\mathbf{r}_{1}\right)$, resulting in a phase modulation with a theoretical contrast of $100 \%$ and a fringe spacing determined by $\lambda / N$. As with path-entangled number states, this corresponds to an $\mathrm{N}$-fold reduced fringe spacing compared to $G^{(1)}(\mathbf{r})$ while keeping a contrast of potentially $100 \%$. Hereby, only tools of linear optics are employed as a single photon is registered at each detector.

To understand this outcome let us consider $N$ identical two-level atoms excited by a single laser $\pi$ pulse. After the spontaneous emission the $N$ photons are registered by $N$ detectors at positions $\mathbf{r}_{1}, \ldots, \mathbf{r}_{N}$. For the sake of simplicity let us consider coincident detection [12]. In that case the $N$ th order correlation function [13] can be written (up to an insignificant prefactor) as [14]

$$
G^{(N)}\left(\mathbf{r}_{1}, \ldots, \mathbf{r}_{N}\right)=\left\langle D^{\dagger}\left(\mathbf{r}_{1}\right) \ldots D^{\dagger}\left(\mathbf{r}_{N}\right) D\left(\mathbf{r}_{N}\right) \ldots D\left(\mathbf{r}_{1}\right)\right\rangle
$$

where

$$
D\left(\mathbf{r}_{i}\right)=\frac{1}{\sqrt{N}} \sum_{\alpha=1}^{N} \sigma_{\alpha}^{-} e^{-i k \mathbf{n}\left(\mathbf{r}_{i}\right) \cdot \mathbf{r}_{\alpha}} .
$$

Here $\mathbf{n}\left(\mathbf{r}_{i}\right)=\mathbf{r}_{i} / r_{i}$ stands for the unit vector in the direction of detector $i$; the sum is over all atom positions $\mathbf{R}_{\alpha}$, $k=\omega_{0} / c$, with $\omega_{0}$ the transition frequency; and $\sigma_{\alpha}^{-}=$ $|g\rangle_{\alpha}\langle e|$ is the lowering operator of atom $\alpha$ for the transition $|e\rangle \rightarrow|g\rangle$.

For all atoms initially prepared in the excited state $|e\rangle$, we obtain from Eqs. (1) and (2)

$$
G^{(N)}\left(\mathbf{r}_{1}, \ldots, \mathbf{r}_{N}\right)=\frac{1}{N^{N}}\left|\gamma\left(\mathbf{r}_{1}, \ldots, \mathbf{r}_{N}\right)\right|^{2},
$$


where [15]

$$
\gamma\left(\mathbf{r}_{1}, \ldots, \mathbf{r}_{N}\right)=\sum_{\substack{\epsilon_{1}, \ldots, \epsilon_{N}=1 \\ \epsilon_{1} \neq \ldots \neq \neq}}^{N} \prod_{\alpha=1}^{N} e^{-i k \mathbf{n}\left(\mathbf{r}_{\epsilon_{\alpha}}\right) \cdot \mathbf{R}_{\alpha}}
$$

Equations (3) and (4) show that $G^{(N)}\left(\mathbf{r}_{1}, \ldots, \mathbf{r}_{N}\right)$ results from the interference of $N$ ! terms, associated with all possibilities to scatter $N$ photons from $N$ identical atoms, which are subsequently registered by $N$ detectors.

To simplify further calculations let us consider the case of $N$ equidistant atoms. By choosing the origin of the coordinate system in the center of the atomic chain, we have

$$
\mathbf{R}_{\alpha}=j_{\alpha} d \mathbf{u},
$$

with $\mathbf{u}$ the unit vector along the chain axis, $d$ the interatomic spacing, and $j_{\alpha}=-(N-1) / 2, \ldots,(N-1) / 2$ for $\alpha=1, \ldots, N$ (see Fig. 1). By defining

$$
\delta\left(\mathbf{r}_{i}\right)=k d \mathbf{n}\left(\mathbf{r}_{i}\right) \cdot \mathbf{u}=k d \sin \theta_{i},
$$

where $\theta_{i}$ is the angle between $\mathbf{n}\left(\mathbf{r}_{i}\right)$ and the direction normal to the atomic chain (see Fig. 1), we find

$$
G^{(N)}\left(\mathbf{r}_{1}, \ldots, \mathbf{r}_{N}\right)=\frac{1}{N^{N}}\left[\sum \cos (\mathbf{j} \cdot \boldsymbol{\delta})\right]^{2} .
$$

Here, $\mathbf{j}$ is the vector of the distances of the atoms from the origin in units of $d$ :

$$
\mathbf{j}=\left(j_{1}, \ldots, j_{N}\right),
$$

$\boldsymbol{\delta}$ is given by

$$
\boldsymbol{\delta}=\left(\delta\left(\mathbf{r}_{1}\right), \ldots, \delta\left(\mathbf{r}_{N}\right)\right),
$$

and the sum in Eq. (7) is over the $N$ ! permutations of the $\mathbf{j}$ components.

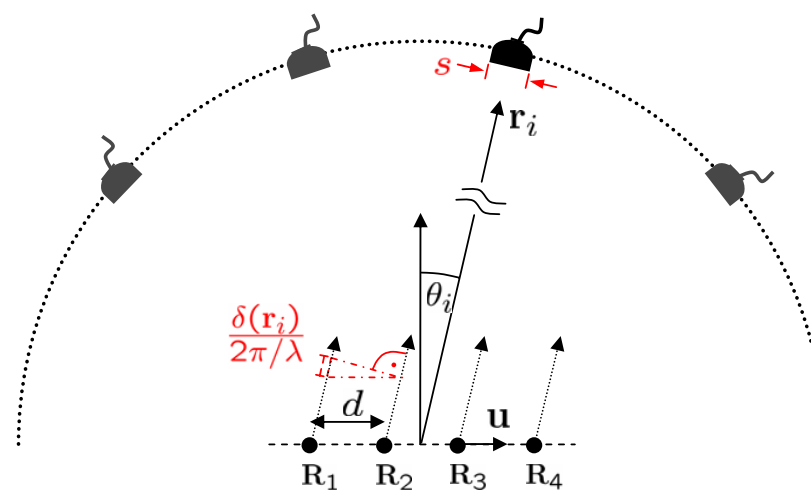

FIG. 1 (color online). Atomic arrangement and detection scheme: $N$ identical two-level atoms at $\mathbf{R}_{1}, \ldots, \mathbf{R}_{N}$ spontaneously emit $N$ photons after excitation by a laser pulse. The photons are recorded in the far field by $N$ detectors positioned at $\mathbf{r}_{1}, \ldots, \mathbf{r}_{N}$. The figure exemplifies the case $N=4$ (for additional symbols see text).
Because of the symmetry of the configuration, the function $G^{(N)}\left(\mathbf{r}_{1}, \ldots, \mathbf{r}_{N}\right)$ contains $N ! / 2$ cosine terms, each oscillating in general with a different spatial frequency. Obviously, the complexity of the expression rises rapidly with increasing atom number $N$. However, if the $N$ detectors are placed in such a manner that all terms in Eq. (7) interfere to give a single cosine, one is left with a modulation oscillating at a unique spatial frequency. This occurs in the following case: for arbitrary even $N$ and choosing the detector positions such that

$$
\begin{aligned}
& \delta\left(\mathbf{r}_{2}\right)=-\delta\left(\mathbf{r}_{1}\right), \\
& \delta\left(\mathbf{r}_{3}\right)=\delta\left(\mathbf{r}_{5}\right)=\ldots=\delta\left(\mathbf{r}_{N-1}\right)=\frac{2 \pi}{N}, \\
& \delta\left(\mathbf{r}_{4}\right)=\delta\left(\mathbf{r}_{6}\right)=\ldots=\delta\left(\mathbf{r}_{N}\right)=-\frac{2 \pi}{N},
\end{aligned}
$$

the $N$ th order correlation function $G^{(N)}$ as a function of detector position $\mathbf{r}_{1}$ reduces to

$$
G^{(N)}\left(\mathbf{r}_{1}\right)=A_{N}\left\{1+\cos \left[N \delta\left(\mathbf{r}_{1}\right)\right]\right\},
$$

where $A_{N}$ is a constant which depends on $N$. For arbitrary odd $N>1$, and choosing the detector positions such that

$$
\begin{aligned}
& \delta\left(\mathbf{r}_{2}\right)=-\delta\left(\mathbf{r}_{1}\right) \\
& \delta\left(\mathbf{r}_{3}\right)=\delta\left(\mathbf{r}_{5}\right)=\ldots=\delta\left(\mathbf{r}_{N}\right)=\frac{2 \pi}{N+1} \\
& \delta\left(\mathbf{r}_{4}\right)=\delta\left(\mathbf{r}_{6}\right)=\ldots=\delta\left(\mathbf{r}_{N-1}\right)=-\frac{2 \pi}{N+1}
\end{aligned}
$$

the $N$ th order correlation function $G^{(N)}$ as a function of $\mathbf{r}_{1}$ reduces to

$$
G^{(N)}\left(\mathbf{r}_{1}\right)=A_{N}\left\{1+\cos \left[(N+1) \delta\left(\mathbf{r}_{1}\right)\right]\right\} .
$$

According to Eqs. (11) and (13), a correlation signal with a modulation of a single cosine can be obtained for any $N$, displaying a contrast of $100 \%$ and a fringe spacing determined by $\lambda / N[\lambda /(N+1)]$ for even [odd] $N$. Note that due to the limited detector sizes and the dipole emission pattern of the spontaneously emitted photons only a subset of all emitted photons will be recorded. However, in contrast to using maximally path-entangled $\mathrm{N}$-photon states we are able to avoid in this scheme both the necessity to generate a state of the form $|\psi(N)\rangle$ and the need to detect a multiphoton absorption signal [16]. We emphasize that as the photons are produced by spontaneous decay the interference signal is generated by incoherent light. We stress further that a fringe contrast implied by Eq. (11) or Eq. (13) proves the underlying quantum nature of the process [1719]. The quantum character is generated by the measurement process after the detection of the first photon. In fact, just before the detection of the $N$ th photon, the atomic system is in an $N$-particle $W$ state with one excitation [20]. The nonclassical characteristics of our scheme are thus 
another example of detection induced entanglement of initially uncorrelated distant particles [19,21-26].

To exemplify our method, let us consider the simplest situation, i.e., the case of $N=2$ atoms. With $\mathbf{j}=$ $\left(-\frac{1}{2},+\frac{1}{2}\right)$ we obtain from Eq. (7)

$$
G^{(2)}\left(\mathbf{r}_{1}, \mathbf{r}_{2}\right)=\frac{1}{2}\left\{1+\cos \left[\delta\left(\mathbf{r}_{1}\right)-\delta\left(\mathbf{r}_{2}\right)\right]\right\} .
$$

Obviously, the modulation of the $G^{(2)}\left(\mathbf{r}_{1}, \mathbf{r}_{2}\right)$ function depends on the relative position of the two detectors (see Fig. 2): for $\delta\left(\mathbf{r}_{2}\right)=\delta\left(\mathbf{r}_{1}\right)$ the second order correlation function is a constant, whereas for fixed $\delta\left(\mathbf{r}_{2}\right)$ the two photon coincidence as a function of $\delta\left(\mathbf{r}_{1}\right)$ exhibits the same phase modulation and fringe spacing as $G^{(1)}(\mathbf{r})$ in Young's double slit experiment. However, the increased parameter space available for the detector positions in case of two detectors allows also to pick out the relative orientation $\delta\left(\mathbf{r}_{2}\right)=-\delta\left(\mathbf{r}_{1}\right)$. In this case we get

$$
G^{(2)}\left(\mathbf{r}_{1}\right)=\frac{1}{2}\left\{1+\cos \left[2 \delta\left(\mathbf{r}_{1}\right)\right]\right\},
$$

exhibiting a phase modulation as a function of $\mathbf{r}_{1}$ with half the fringe spacing of $G^{(1)}(\mathbf{r})$ while keeping a contrast of $100 \%$ (see also [15]). Note that the assumed condition for the direction of emission of the two photons, i.e., $\delta\left(\mathbf{r}_{2}\right)=$ $-\delta\left(\mathbf{r}_{1}\right)$, corresponds to a space-momentum correlation of

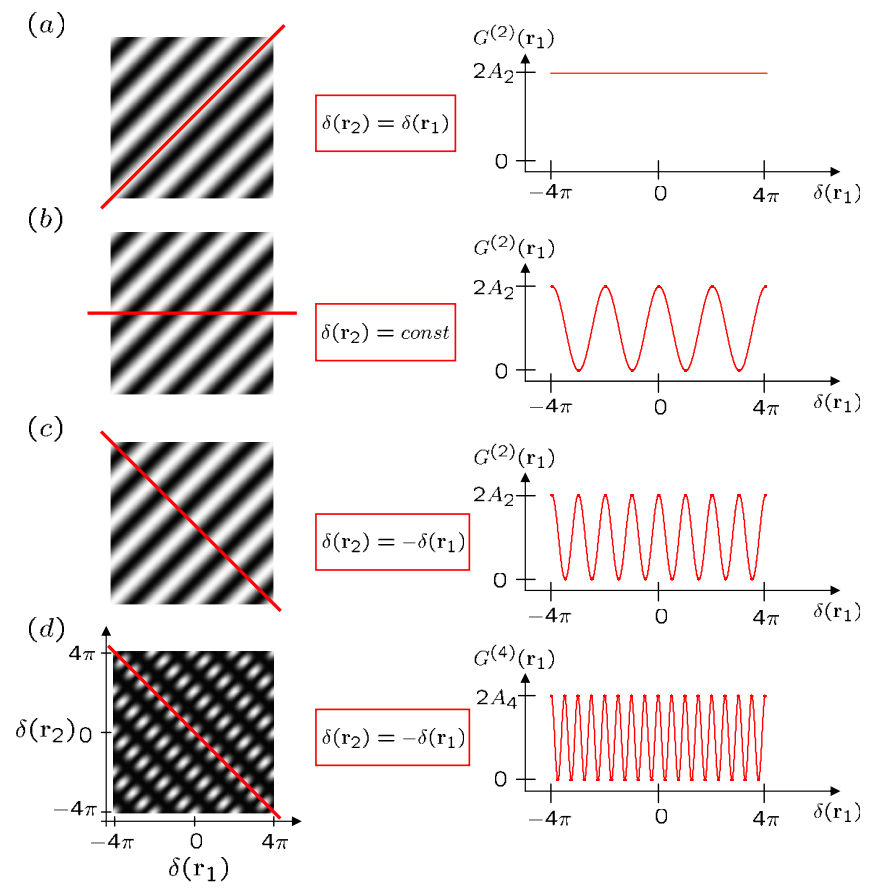

FIG. 2 (color online). Left (a)-(c): density plots of $G^{(2)}\left(\mathbf{r}_{1}, \mathbf{r}_{2}\right)$ for two atoms versus $\delta\left(\mathbf{r}_{1}\right)$ and $\delta\left(\mathbf{r}_{2}\right)$; left (d): density plot of $G^{(4)}\left(\mathbf{r}_{1}, \mathbf{r}_{2}, \mathbf{r}_{3}, \mathbf{r}_{4}\right)$ for four atoms versus $\delta\left(\mathbf{r}_{1}\right)$ and $\delta\left(\mathbf{r}_{2}\right)$, with $\delta\left(\mathbf{r}_{3}\right)=\pi / 2$ and $\delta\left(\mathbf{r}_{4}\right)=-\pi / 2$. Right: cuts through the density plots along the indicated lines, i.e., for (a) $\delta\left(\mathbf{r}_{2}\right)=\delta\left(\mathbf{r}_{1}\right)$, (b) $\delta\left(\mathbf{r}_{2}\right)=$ const, and (c),(d) $\delta\left(\mathbf{r}_{2}\right)=-\delta\left(\mathbf{r}_{1}\right)$. the photons identical to the one present in spontaneous parametric down-conversion [5,6,27].

In the case of the fourth order correlation function $G^{(4)}\left(\mathbf{r}_{1}, \mathbf{r}_{2}, \mathbf{r}_{3}, \mathbf{r}_{4}\right)$ for four equidistant atoms, and by placing the detectors according to Eq. (10) (see Fig. 2), one finds

$$
G^{(4)}\left(\mathbf{r}_{1}\right)=\frac{1}{8}\left\{1+\cos \left[4 \delta\left(\mathbf{r}_{1}\right)\right]\right\} .
$$

Obviously, $G^{(4)}$ as a function of $\mathbf{r}_{1}$ exhibits a modulation of a single cosine with a contrast of $100 \%$, in this case with a fringe spacing determined by $\lambda / 4$.

As an example, let us apply our scheme in the context of microscopy. From Abbe's theory of the microscope we know that an object can be resolved only if at least two principal maxima of the diffraction pattern are included in the image formation [1]. According to this criterion the use of the first order correlation function $G^{(1)}\left(\mathbf{r}_{1}\right)$ for imaging $N$ equidistant atoms allows at best to resolve an interatomic spacing equal to $\lambda$ [1]. Indeed, if each atom is initially prepared in the state $|\phi\rangle=\frac{1}{\sqrt{2}}(|g\rangle+|e\rangle)$, we get from Eqs. (1) and (2)

$$
G^{(1)}\left(\mathbf{r}_{1}\right)=\frac{1}{2}\left\{1+\frac{1}{N} \sum_{\alpha=1}^{N-1}(N-\alpha) \cos \left[\alpha \delta\left(\mathbf{r}_{1}\right)\right]\right\} .
$$

Equation (17) equals (up to an offset) the outcome of the classical grating. As is well-known from the grating equation [and as Eq. (17) explicitly shows] two principal maxima appear in the far-field diffraction pattern only if the

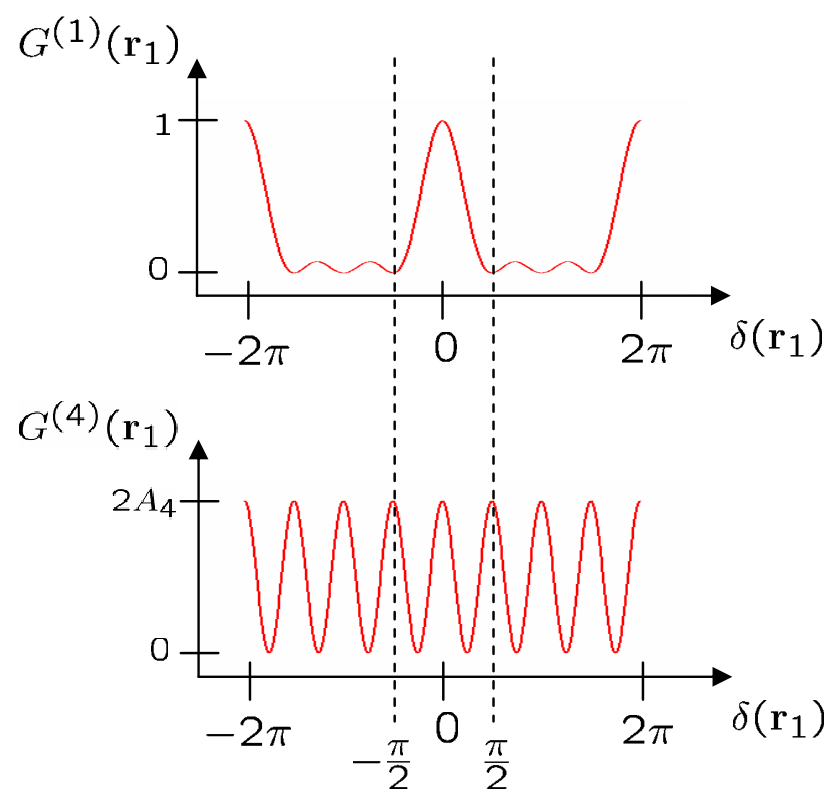

FIG. 3 (color online). $G^{(1)}\left(\mathbf{r}_{1}\right)$ and $G^{(4)}\left(\mathbf{r}_{1}\right)$ as a function of $\delta\left(\mathbf{r}_{1}\right)$ for a chain of 4 atoms. The interval $[-2 \pi, 2 \pi]$ corresponds to the maximal range of variation of $\delta\left(\mathbf{r}_{1}\right)$ for an interatomic distance $d=\lambda$ [see Eq. (6)]. The dashed lines indicate the corresponding range in case of $d=\lambda / 4$. 
interatomic distance is greater or equal to $\lambda$ (see Fig. 3). By contrast, the use of the $N$ th order correlation function with the $N$ detectors positioned according to Eq. (10) [or Eq. (12)] allows to resolve an atom-atom separation as small as $\lambda / N$ [or $\lambda /(N+1)$ ] (see Fig. 3). In this way the $N$ th order correlation function $G^{(N)}\left(\mathbf{r}_{1}\right)$ can be used to resolve and image trapped atoms separated by a distance $d=\lambda / N$.

Finally, we address the technical feasibility of our scheme. For the ability to localize atoms and adequately resolve optical path differences on a scale smaller than $\lambda$ we refer to [28-30]. A detector of a given size $s$ positioned at a distance $L=\left|\mathbf{r}_{i}\right|$ in the far-field region (see Fig. 1) gives rise to an angular resolution $\Delta \theta=s / L$, i.e., to a phase resolution $\Delta \delta=k d \cos \theta \Delta \theta$. To resolve the modulation of the $N$ th order correlation function $G^{(N)}\left(\mathbf{r}_{1}\right)$, a sufficient requirement is that $N \Delta \delta \ll 2 \pi$, i.e., $\Delta \theta \ll$ $\lambda /(N d)$, which yields the condition

$$
L \gg s \frac{N d}{\lambda} \text {. }
$$

For given $N$ and $d$ we can thus find for any detector size $s$ a distance $L$ to achieve the necessary resolution. Hereby, choosing the smallest $L$ compatible with Eq. (18) is favorable in order to maximize the $\mathrm{N}$-photon detection probability; the exact longitudinal positions of the detectors are thereby not important. In case of a gaussian distribution of the phases $\delta\left(\mathbf{r}_{i}\right)(i=2, \ldots, N)$ around their ideal values given by Eqs. (10) or (12) with a standard deviation $\sigma$ the contrast of the $G^{(N)}\left(\mathbf{r}_{1}\right)$ function is reduced and given by $e^{-N \sigma^{2} / 4}$. For $N=2$ and $N=4$, this means that a contrast of higher than $50 \%$ can be maintained as long as $\sigma$ is less than 0.8 and 1.2, respectively. Using the set of reasonable parameters $d=5 \mu \mathrm{m}, \quad \Delta d=0.1 \mu \mathrm{m}, \quad \theta\left(\mathbf{r}_{1}\right)=30^{\circ}$, $\Delta \theta\left(\mathbf{r}_{1}\right)=0.1^{\circ}, k=2 \pi / 800 \mathrm{~nm}$, and $\Delta k<10^{-7} k$ we obtain $\sigma \approx 0.7$.

In conclusion we have shown that $N$ photons of wavelength $\lambda$ spontaneously emitted by $N$ atoms and coincidentally recorded by $N$ detectors at particular positions exhibit correlations and interference properties similar to classical coherent light of wavelength $\lambda / N$. The method requires neither initially entangled states nor multiphoton absorption, only common single-photon detectors.

We gratefully acknowledge financial support by the Dr. Hertha und Helmut Schmauser foundation. G. S. A. thanks NSF Grant No. NSF-CCF-0524673 for supporting this collaboration.

*jvz@optik.uni-erlangen.de; http://www.optik.uni-erlangen.de/jvz/
[1] M. Born and E. Wolf, Principles of Optics (Pergamon Press, New York, 1980).

[2] E. J. S. Fonseca, C. H. Monken, and S. Pàdua, Phys. Rev. Lett. 82, 2868 (1999).

[3] K. Edamatsu, R. Shimizu, and T. Itoh, Phys. Rev. Lett. 89, 213601 (2002).

[4] A. N. Boto et al., Phys. Rev. Lett. 85, 2733 (2000).

[5] M. D'Angelo, M. V. Chekhova, and Y. Shih, Phys. Rev. Lett. 87, 013602 (2001).

[6] R. S. Bennink et al., Phys. Rev. Lett. 92, 033601 (2004).

[7] P. Walther et al., Nature (London) 429, 158 (2004); M. W. Mitchell, J. S. Lundeen, and A. M. Steinberg, Nature (London) 429, 161 (2004); Z. Zhao et al., Nature (London) 430, 54 (2004).

[8] A. Muthukrishnan, M. O. Scully, and M.S. Zubairy, J. Opt. B 6, S575 (2004).

[9] J. J. Bollinger, W. M. Itano, D. J. Wineland, and D. J. Heinzen, Phys. Rev. A 54, R4649 (1996).

[10] G. S. Agarwal and M. O. Scully, Opt. Lett. 28, 462 (2003).

[11] More recently, alternative schemes have been proposed using classical light to achieve subwavelength resolution. These approaches avoid creation of entangled states but require multiphoton detection and/or generate an image with reduced visibility. See S. J. Bentley and R. W. Boyd, Opt. Express 12, 5735 (2004); A. Peer et al., Opt. Express 12, 6600 (2004); K. Wang and D.-Z. Cao, Phys. Rev. A 70, 041801(R) (2004); P. R. Hemmer et al., Phys. Rev. Lett. 96, 163603 (2006).

[12] We emphasize that coincident detection is not a prerequisite for our scheme. The requirement is that all photons emitted by the $N$ atoms are recorded by the $N$ detectors; the exact detection time does not influence the contrast nor the resolution of the correlation signal.

[13] R. J. Glauber, Phys. Rev. 130, 2529 (1963).

[14] G.S. Agarwal, Quantum Optics, Springer Tracts in Modern Physics Vol. 70 (Springer, Berlin, 1974).

[15] G. S. Agarwal et al., Phys. Rev. A 70, 063816 (2004).

[16] Note that conditions (10) and (12) do not mean that the different detectors have to be at the same place, since equality of phase shifts does not mean equality of detector positions.

[17] L. Mandel, Phys. Rev. A 28, 929 (1983).

[18] Z. Y. Ou, Phys. Rev. A 37, 1607 (1988).

[19] C. Skornia et al., Phys. Rev. A 64, 063801 (2001).

[20] W. Dür, G. Vidal, and J. I. Cirac, Phys. Rev. A 62, 062314 (2000).

[21] C. Cabrillo et al., Phys. Rev. A 59, 1025 (1999).

[22] S. Bose et al., Phys. Rev. Lett. 83, 5158 (1999).

[23] E. S. Polzik, Phys. Rev. A 59, 4202 (1999).

[24] C. W. Chou et al., Nature (London) 438, 828 (2005).

[25] J. Beugnon et al., Nature (London) 440, 779 (2006).

[26] P. Maunz et al., arXiv:quant-ph/0608047.

[27] Y. Shih, Eur. Phys. J. D 22, 485 (2003).

[28] U. Eichmann et al., Phys. Rev. Lett. 70, 2359 (1993).

[29] Y. Miroshnychenko et al., Nature (London) 442, 151 (2006).

[30] D. Stick et al., Nature Phys. 2, 36 (2006). 